\title{
BitClust: fast geometrical clustering of long molecular dynamics simulations
}

Roy González-Alemán ${ }^{\dagger *}$, David Hernández-Castillo ${ }^{\dagger}$, Alejandro Rodríguez-Serradet ${ }^{\dagger}$, Julio Caballero $^{+}$, Erix W. Hernández-Rodríguez ${ }^{\phi}$, Luis Montero-Cabrera ${ }^{\dagger}$

†Laboratorio de Química Computacional y Teórica, Facultad de Química, Universidad de La Habana, Zapata y G, Vedado 10400, La Habana, Cuba.

† Centro de Bioinformática y Simulación Molecular, Facultad de Ingeniería en Bioinformática, Universidad de Talca, 2 Norte 685, Casilla 721, Talca, Chile.

${ }^{\Phi}$ Escuela de Química y Farmacia, Facultad de Medicina, Universidad Católica del Maule, 3460000 Talca, Chile.

E-mail: roy_gonzalez@,fq.uh.cu 
Section S1: Details about generation of analyzed trajectories.

\section{$\underline{6 K}$}

All details on generation of $6 \mathrm{~K}$ trajectory has been previously published (Shea, J. E., \& Levine, Z. A. (2016). Studying the early stages of protein aggregation using replica exchange molecular dynamics simulations. In Protein Amyloid Aggregation (pp. 225-250). Humana Press, New York, NY)

\section{$\underline{100 K}$}

The 2.2 A X-ray bovine-rhodopsin structure (Protein Data Bank code: 1U19) inside a palmitoyl-oleoyl-phosphatidylcholine (POPC) hydrated membrane. The protonation states of the titratable amino-acid residues were assigned according to the protocol described by Okada et. al. ${ }^{1}$ The internal water molecules were conserved in the rhodopsin model. The rhodopsin/POPC/water system was neutralized at near-physiological concentration $(150 \mathrm{mM})$ using $\mathrm{Na}^{+}$and $\mathrm{Cl}^{-}$ions, where at least $15.0 \AA$ of space was left between the rhodopsin structure and cell boundaries. The TIP3P model ${ }^{3}$ was used for all water molecules. The two palmitoyl residues covalently linked to the residues Cys322 and Cys323 were kept in the three-dimensional structure, as well as, the Cys110-Cys187 disulfide bridge.

A 100-ns MD trajectory was computed with the NAMD 2.12 ${ }^{4}$ program, using the CHARMM36 force field ${ }^{5,6}$. The system was simulated at the constant temperature of $310 \mathrm{~K}$ and pressure of 1.0 bar by using the Nosé-Hoover thermostat and barostat ${ }^{7}$. A timestep of 1.0 fs was used for the production 100-ns run, and the frames were saved every $1 \mathrm{ps,}$, which allowed to obtain a MD trajectory containing 100.000 frames $(100 \mathrm{~K}$ trajectory, $\mathrm{K}=1.000)$. A cutoff of $12 \AA$ was used to compute the other nonbonded interactions. The system was simulated using periodic boundary 
conditions (PBC), which were considered in the calculations of long-range interactions for the full system, using the particle-mesh Ewald method ${ }^{-}$with a grid resolution less than $1.0 \AA \AA$.

A first minimization-equilibration cycle was performed while both all rhodopsin atoms and internal water molecules were fixed, and the solvating water molecules were relaxed by a 10.000step conjugated gradient (CG) minimization, followed by 4-ns NVT MD simulation. In the second minimization-equilibration cycle, the protein backbone atoms were fixed and the system was again subjected to a 10.000-step CG minimization, followed by 4-ns NPT simulation. Subsequently, a third 8-ns NPT equilibration cycle was performed with the protein free to move. Finally, a production 100-ns NPT run was performed without applying any restraint to obtain the $100 \mathrm{~K}$ trajectory. The resulting geometries were visualized using VMD 1.9.3 ${ }^{9}$. The root-mean-square deviation of the secondary structure backbone of the rhodopsin embedded inside the hydrated lipid bilayer was used to assess the stability of the protein structure during the MD simulation. The rhodopsin structure is stable along the $100 \mathrm{~K}$ trajectory.

\section{$\underline{500 K}$}

To generate the long trajectory $500 \mathrm{~K}$ we randomly combined 100,000 frames from the $6 \mathrm{~K}$ trajectory and then we concatenated five times those 100,000 frames.

Table S1: Example of bitwise operations AND and XOR applied to bit vectors A and B.

\begin{tabular}{lllll}
\hline A & 0 & 0 & 1 & 1 \\
\hline B & 0 & 1 & 0 & 1 \\
\hline A AND B & 0 & 0 & 0 & 1
\end{tabular}


A XOR B $\quad 0 \quad 1 \quad 1 \quad 0$

\section{Section S2: Availability, installation and basic usage}

Before installing BitClust in your system, please be sure to have the MDTraj code available. BitClust is available at PYPI (https://pypi.org/project/bitclust/) and GitHub (https://github.com/rglez/bitclust/) under the GNU General Public License v3.0. To install it from PYPI, just type pip install bitclust from a terminal in Windows or Linux operative systems. To install it from GitHub, first download and extract the project file and once inside the main directory, type python setup.py install. For more specific details on installation, you can refer to the project documentation (https://bitclust.readthedocs.io/en/latest/index.html).

Once installed, running bitclust $-\boldsymbol{h}$ in a terminal give access to the program's help which contains short descriptions of available arguments. BitClust code inherits acceptable trajectory and topology formats from MDTraj as well as the selection syntax. Formats include those generated by the main standard software (CHARMM, GROMACS, AMBER, and NAMD) while the selection syntax is very rich and flexible.

Only one argument is always mandatory, -traj, which specifies the path to trajectory file. If trajectory format does not provide topological information of the system, this information must be supplied from a topology file through the -top argument. All other arguments are always optional and if not explicitly specified, they take default values.

A minimal run like bitclust -top tau_6k.pdb-traj tau_6k.dcd loads the tau_6k.ded trajectory into tau_6k.pdb coordinates, both present on the current working directory, and performs a clustering job using Daura's algorithm with a cutoff of 1A (-cutoff 1$)$ on the whole trajectory. Use -first, - 
last and -stride to select an interval (they defaults to 0, last and 1, respectively). System selection defaults to all atoms (-sel all) and BitClust will retrieve all clusters with at least 2 frames (-size 2). Frame 0 will be used as reference (-ref 0$)$ to make an RMSD graph and all produced output will be saved in the current working directory (-odir ./*).

BitClust outputs basic graphics for fast inspection of the clustering job results (see SI-4). All of them and others can be constructed from two generated text files: clusters_statistics.txt and frames_statistics.txt. The first one contains as columns every cluster ID (starting from $0,-1$ corresponds to unclustered frames), cluster size, cluster size percent calculated from the total of frames and the center frame of every cluster. The second file contains as columns every frame ID (starting from 0), the cluster ID where every frame belongs to and the RMSD value of every frame respect to the specified reference (default reference is frame 0 ). 

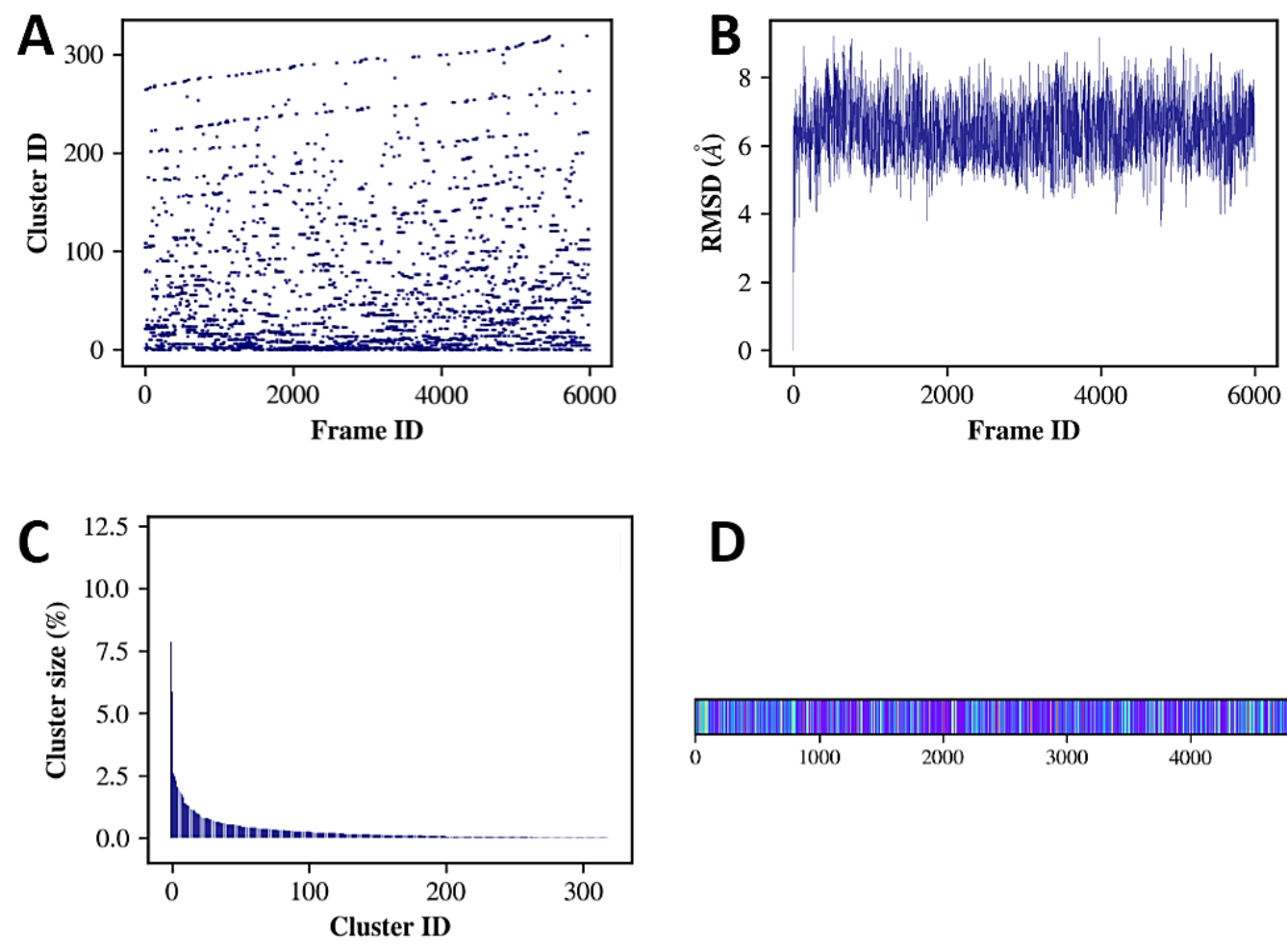

D

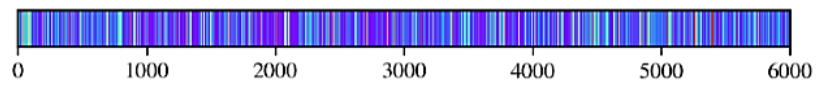

Figure-S1: Automatic graphics generated by BitClust. These and others are easily constructed from the two output text files. A-) Cluster membership of every frame B-) RMSD of all frames vs. reference frame C-) Size of clusters in percent, and D-) Color bar representing cluster membership of every frame

\section{REFERENCES}

1. Okada, T.; Sugihara, M.; Bondar, A. N.; Elstner, M.; Entel, P.; Buss, V. J Mol Biol 2004, $342(2), 571-583$.

2. Protein Data Bank; see www.pdb.org.

3. Jorgensen, W. L.; Chandrasekhar, J.; Madura, J. D.; Impey, R. W.; Klein, M. L. J Chem Phys 1983, 79, 926-928. 
4. Phillips, J. C.; Braun, R.; Wang, W.; Gumbart, J.; Tajkhorshid, E.; Villa, E.; Chipot, C.; Skeel, R. D.; Kale, L.; Schulten, K. J Comput Chem 2005, 26(16), 1781-1802.

5. MacKerell, A. D., Jr.; Bashford, D.; Bellott, M.; Dunbrack, R. L.; Evanseck, J. D.; Field, M. J.; Fischer, S.; Gao, J.; Guo, H.; Ha, S.; Joseph-McCarthy, D.; Kuchnir, L.; Kuczera, K.; Lau, F. T. K.; Mattos, C.; Michnick, S.; Ngo, T.; Nguyen, D. T.; Prodhom, B.; Reiher, W. E., III; Roux, B.; Schlenkrich, M.; Smith, J. C.; Stote, R.; Straub, J.; Watanabe, M.; Wiorkiewicz-Kuczera, J.; Yin, D.; Karplus, M. J Phys Chem B 1998, 102(18), 3586-3616.

6. MacKerell, A. D., Jr.; Feig, M.; Brooks, C. L., III. J Comput Chem 2004, 25(11), 14001415.

7. Nosé, S. J Chem Phys 1984, 81(1), 511-519.

8. Essmann, U.; Perera, L.; Berkowitz, M. L.; Darden, T.; Lee, H.; Pedersen, L. G. J Chem Phys 1995, 103(19), 8577-8593.

9. Humphrey, W.; Dalke, A.; Schulten, K. J Mol Graphics 1996, 14, 33-38. 